\title{
QUALITY IMPROVEMENT Assessment of serum calcium in patients referred for suspected lung cancer: A quality improvement project to enhance patient safety in clinical practice
}

\author{
Authors: Christina Apthorp, ${ }^{\mathrm{A}}$ Sagana Kirisnathas, ${ }^{\mathrm{B}}$ Nikolaos Stavrakas, ${ }^{\mathrm{C}}$ Isuru Warakagoda, ${ }^{\mathrm{D}}$ Stephen Crooks $^{\mathrm{E}}$ and \\ Jayanta Mukherjee ${ }^{\mathrm{E}}$
}

\section{Background}

Hypercalcaemia is a serious complication of lung cancer. A quality improvement project (QIP) was designed based on guidance from the American College of Chest Physician and the European Respiratory Society who recommend measuring serum calcium for patients referred with suspected lung cancer.

Method

Seventy-two patients were included in the initial data to ascertain the delay between referral to the lung cancer pathway and obtaining serum calcium levels as part of the initial work-up. New data were then collected after each intervention (including presentations at weekly respiratory multidisciplinary team meetings, posters within clinical areas and a hospital trust screensaver) to evaluate the delay.

Results

Initially, $11.1 \%(n=8)$ did not have serum calcium measured at any point; two of which had lung cancer (including one metastatic malignancy). Of those who had serum calcium measured, there was a median delay of 13 days between first suspicion and obtaining serum calcium. After all the interventions were put in place, patients had a median of 7 days' delay $(p=0.001)$.

Conclusion

This QIP design was based on continued feedback to improve the care of patients suspected of lung cancer. Although there was a significant reduction in delays post-intervention, increasing awareness in the community is suggested to maintain these improvements.

KEYWORDS: lung, respiratory, cancer, oncology, quality improvement project

DOI: $10.7861 /$ fhj.2020-0068

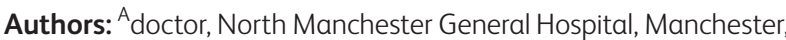
UK; ${ }^{B}$ doctor, Croydon University Hospital, Croydon, UK; ${ }^{\text {C doctor, }}$ Norfolk and Norwich University Hospital, Norwich, UK; ' doctor,

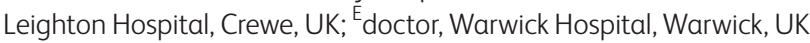

\section{Introduction}

It is well recognised that elevated serum calcium is a serious complication of lung cancer, with known aetiology of three distinct mechanisms: PTHrP mediated humoral hypercalcaemia, dysregulated calcitriol production and local osteolytic hypercalcaemia. $^{1}$

Tumours associated with hypercalcaemia are reported to exhibit poorer prognosis. Stewart reported that $\sim 50 \%$ of patients die within 30 days after the detection of hypercalcaemia, highlighting the importance of early detection of hypercalcaemia and active treatment in such cases. ${ }^{2}$

The American College of Chest Physicians and the European Respiratory Society recommend urea and electrolytes, blood count, liver function and serum calcium tests in all patients referred with suspected lung cancer. ${ }^{3,4}$ However, the National Institute for Health and Care Excellence (NICE) provides no advice to instruct the requirement of determining serum calcium levels or equally any other blood investigations in the workup of lung cancer. ${ }^{5}$ Clinical experience from respiratory physicians in the UK, suggests that testing for serum calcium levels are often omitted. This may be due to the lack of guidelines from British sources.

The aim of this quality improvement project (QIP) is to reduce the delay between initial suspicion of lung cancer and serum calcium level measured as part of their initial workup to improve patient outcomes.

\section{Methods}

Patients were accepted to this QIP retrospectively from 5 consecutive weekly lung multidisciplinary team (MDT) meetings, with formal ethical approval from the trust. One-hundred patients were initially identified from this data in March 2018 for the initial study. However, after excluding patients with duplicate records, benign disease and those who decided to continue their care through the private sector, 72 patients were investigated initially. ${ }^{6}$

The data required for this study were collected via the hospital's confidential e-records system, obtaining the date of initial referral to the lung cancer team (LCT) and subsequently the adjusted 
calcium and alanine aminotransferase (ALT) levels. ALT was used as a standard blood test. The time between a standard blood test, ALT, and an adjusted serum calcium blood test was compared. Patients referred to the LCT with calcium and ALT checked within 4 weeks prior to the initial suspicion of lung cancer were accepted to the study and were recorded to have a 0 -day interval between initial referral and blood investigations, this would take into account the cases in which calcium levels were deliberately not requested because of availability of current levels. For the initial study, data were collected up to 1 year after the final lung cancer MDT meeting.

Postcodes were recorded to aid the removal of data from patients who would be receiving treatment from outside the trust catchment area, as access to these records would not be available and therefore could not be considered in the study.

Interventions focused on the secondary care setting were conducted over a period of 9 months to evaluate which intervention would have the largest impact. From March 2019, the findings were presented weekly at the MDT meetings by a respiratory consultant to encourage the respiratory department's awareness and participation in ordering calcium as part of the initial workup for patients with suspected lung cancer. In September 2019, posters designed by the team were displayed around the hospital in all clinical areas, highlighting the initial results and urging all physicians to also consider ordering serum calcium levels as part of their initial workup. Furthermore, in November 2019, trust hospital computers were also used to display data of the initial study via the weekly screensaver bulletin. This intervention was chosen as a platform to further encourage all physicians within the trust to order serum calcium levels when referring patients with a suspicion of lung malignancy.

Post-intervention data were analysed, including 94 patients postMDT presentation, 55 following posters being displayed around the hospital and 21 following weekly screensaver bulletins.

Following each intervention, the median delay was calculated from the first date of suspected lung cancer and to the date of the obtained serum calcium level, as per the method of the initial data. These results were used to evaluate the effectiveness of the interventions made.

\section{Results}

Fig 1 summarises the outline of the QIP.

Initial data revealed that eight out of 72 patients did not have serum calcium checked at any point within the pathway (Fig 2a): two had lung cancer (one with metastatic M1b), one remains under surveillance for suspicious nodules and five had no malignancy and were discharged. Thirteen patients did not have their calcium measured within 30 days of initial referral (range 35-573). Within the first cohort of 72 patients, a median delay of 13 days was calculated between the first suspicion of lung cancer and the request of serum calcium. The date of serum calcium investigations was initially compared to the ALT blood results. ALT results had a median of 0 days' delay from initial cancer suspicion.

Four out of the $72(5.6 \%)$ patients in this study were recorded to have hypercalcaemia ( $>2.6 \mathrm{mmol} / \mathrm{L})$. One previously diagnosed with primary hyperparathyroidism and three with a new diagnosis of lung cancer. The median survival of these three lung cancer sufferers, from the date of the first detection of hypercalcaemia,

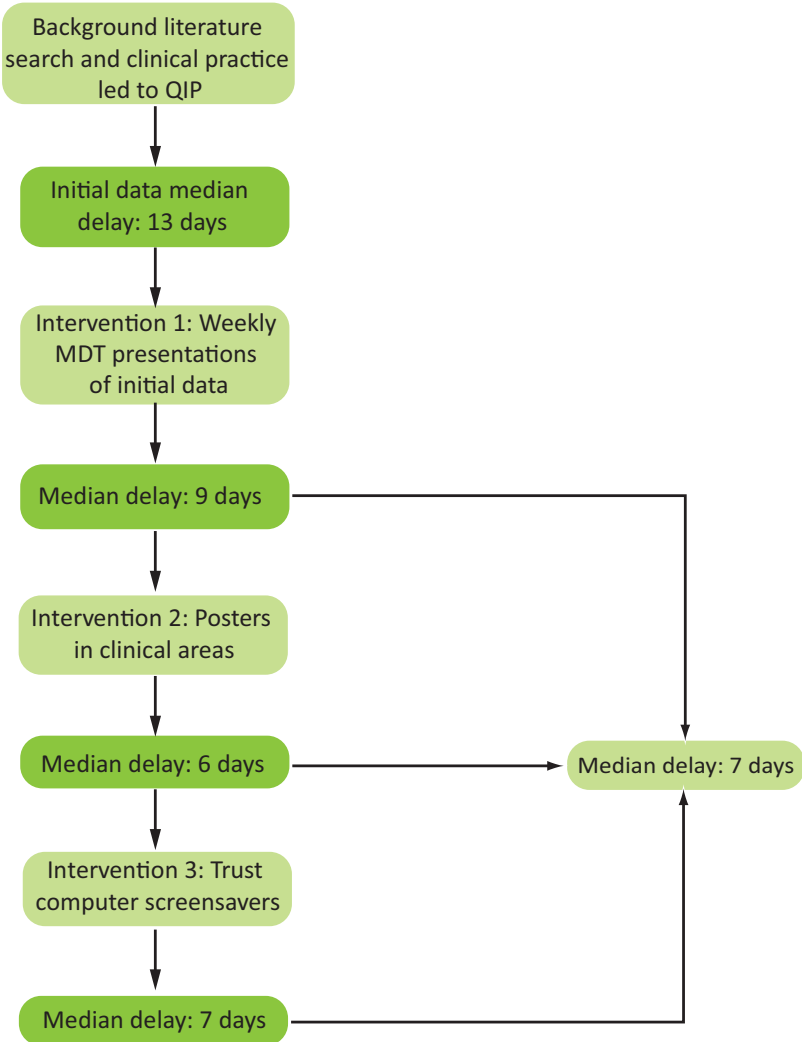

Fig 1. Outline of the quality improvement project showing three interventions and results. MDT = multidisciplinary team; QIP = quality improvement project.

was 39 days (individually 19, 39 and 50 days). Out of the 72 patients in this study, 35 were diagnosed with lung cancer. Other diagnoses included lymphoma, interstitial lung disease and lung as a secondary metastasis.

After each individual intervention, data were recorded regarding the delay in days between the initial suspicion of lung cancer and the date serum calcium was measured (Fig 2b). Following the weekly MDT meetings, a median delay of 9 days was highlighted. Subsequently, the posters displayed around clinical areas within the trust led to a decreased delay of 6 days between suspicion and investigation. Thirdly, presenting the data on the trust screensavers led to a 7-day delay. Conclusively, in patients that had serum calcium measured after the interventions, a median of 7 days' delay between the first suspicion of cancer and obtaining serum calcium was found $(p=0.001)$.

Box plots of the data show a comparison of the effect of the delays pre-intervention and post-intervention (Fig 3).

\section{Discussion}

Eight out of 72 patients referred via the 2-week wait pathway did not have serum calcium measured. Among the remaining 64 patients, there was a median of 13 days' delay between the suspicion of lung cancer and the investigation of serum calcium. This is not the case for liver function tests, which had a median 
a

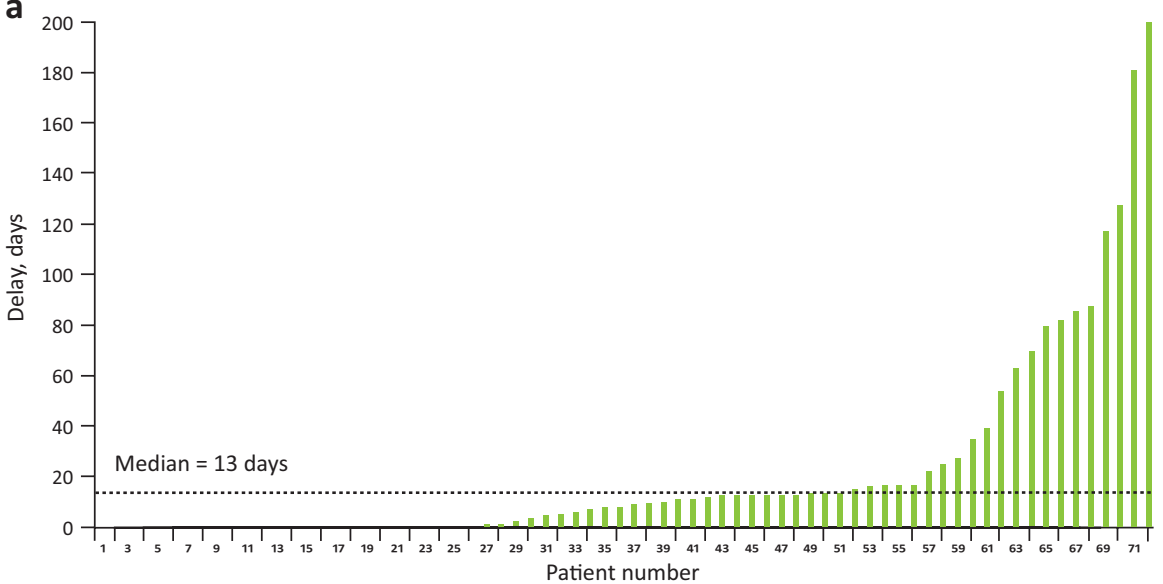

b

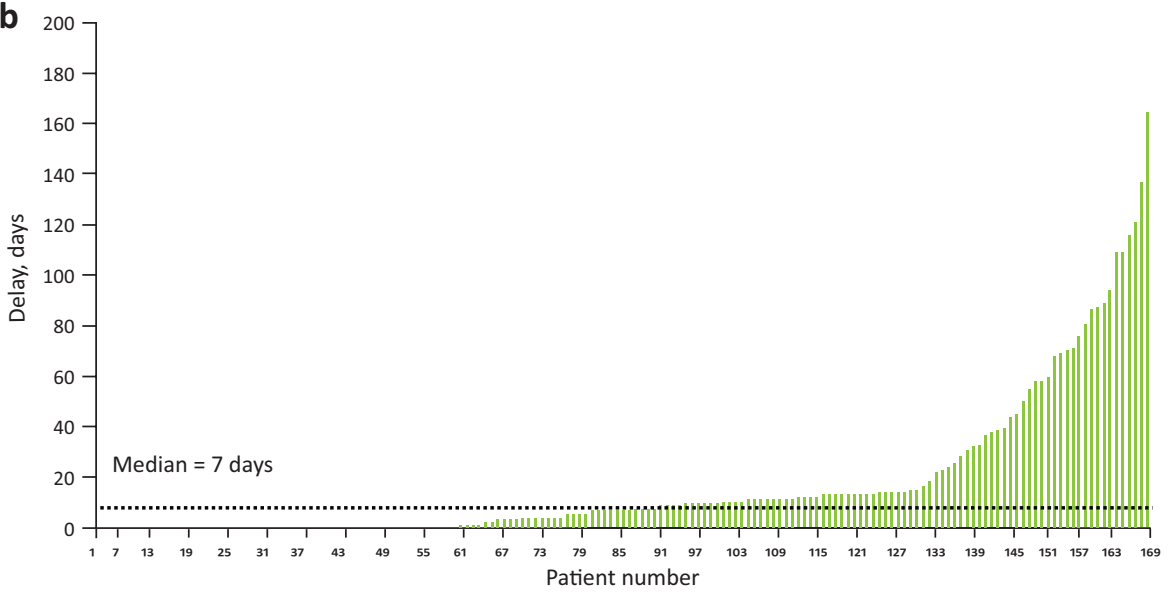

Fig 2. Delays between initial referral to the lung cancer pathway and obtaining serum calcium levels. a) Preintervention. b) Post-intervention. delay of 0 days. New data was subsequently collected after each intervention (including weekly MDT presentations of the initial data, posters targeting healthcare professionals in hospitals and computer trust screensavers) to evaluate the delay. Following intervention in the secondary care setting, there was a median of 0 days' delay. The significant reduction in delays post-intervention indicated the need to increase awareness of measuring serum calcium to improve the care of patients suspected of lung cancer. It could be suggested that a calcium blood test is included as part

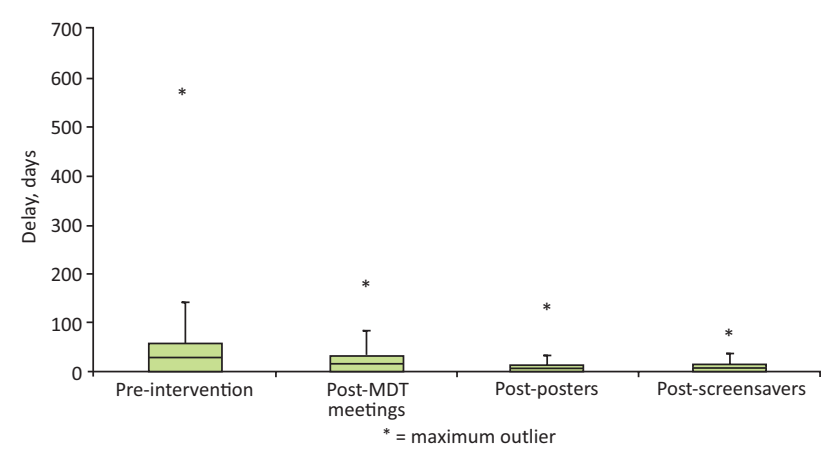

Fig 3. Effect on delays across interventions. MDT = multidisciplinary team. of the initial primary care blood work for patients referred under the 2-week wait pathway.

This QIP demonstrates the effectiveness of the imposed interventions on the median delay between the first suspicion of lung cancer and obtaining serum calcium results. The importance of a recorded high serum calcium was highlighted as all patients $(n=3)$ found to have hypercalcaemia directly linked to their malignancy were recorded to have a median survival of 39 days from the date of hypercalcaemia detection. Improving the median survival of patients by detecting hypercalcaemia early was a key finding of this QIP. To note, it is uncertain whether all raised calcium levels can be attributed to bone metastasis or other malignancy-related pathologies including PTHrP secretion.

The cost-benefit of hypercalcaemia at a population level is useful to understand in terms of preventing emergency admissions, as well as the prevention of secondary fractures from those diagnosed with bone metastases. Hypercalcemia associated with lung cancer can commonly be attributed to bone metastasis, with known issues to mobility and impairment to a patient's quality of life. Bone-related metastasis can subsequently lead to a financial burden on the healthcare system of up to $€ 3,999$, and those with a pathological fracture or spinal cord compression having a much higher financial burden of up to $€ 4,672$ monthly; 
highlighting the link between poor prognosis and the implication of overall treatment cost. ${ }^{7}$

The recommendation is to undertake a prospective study in a small number of general practitioner practices in a region with a high incidence of de novo metastatic lung cancer where hypercalcaemia testing is incorporated early into the diagnostic package vs practices where it is not. This study would be useful to make a conclusion on cost-benefit analysis as there are currently limited data.

All data were obtained from a single source, the MDT list from the quality and data team of the trust. Although this may raise questions, the team decided this would be the most appropriate source as all patients referred via the 2-week wait pathway are discussed at the weekly MDT meeting, therefore all patients referred with suspected lung malignancy would be captured.

The sample size was chosen after eliminating duplicates and patients managed under the private healthcare sector. Ideally, patient information would have been sourced from other hospital trusts, providing a bigger sample size with a large variety in terms of demographics.

The risk of the data being anomalous due to chance was limited by conducting this study over a 21 -month period. The aim was to reduce confounding factors that may have affected the true results. This QIP was well designed, based on continued feedback to improve the prognosis of patients suspected of lung cancer. Although there was a significant reduction in delays post-intervention, results can only be attributed to the one trust, therefore, interventions to a wider demographic would be required with a need to increase awareness of these results in the community, especially among primary care physicians to maintain these improvements and further see a reduction in the number of days in delay.

\section{Conclusion}

This QIP highlights the importance of measuring serum calcium levels at the point of referral to improve patient safety.
Implementing these simple and sustainable steps at a national level will allow for the increased identification of hypercalcaemia, potentially leading to an improvement in prognosis nationally to those suspected of lung cancer.

\section{References}

1 Nemr S, Alluri S, Syndaramurthy D, Landry D, Braden G. Hypercalcaemia in lung cancer due to simultaneously elevated PTHrP and ectopic calcitriol production: first report. Case Reports in Oncological Medicine 2017:2583217.

2 Stewart AF. Clinical practice. Hypercalcemia associated with cancer. N Engl J Med 2005:352:373-9.

3 Ost D, Jim Yeung S, Tanoue $L$ et al. Clinical and organizational factors in the initial evaluation of patients with lung cancer. Chest 2013;143:e121S-41S

4 Detterbeck F, Lewis S, Diekemper R, Addrizzo-Harris D, Alberts W. Executive summary: Diagnosis and management of lung cancer, 3rd ed: American College of Chest Physicians evidence-based clinical practice guidelines. Chest 2013;143:7S-37S.

5 Health National Institute for and Excellence Care. Lung cancer: diagnosis and management: NICE guideline [NG122]. NICE, 2019. www.nice.org.uk/guidance/cg122

6 Apthorp C, Kirisnathas S, Stavrakas N et al. Assessment of serum calcium in patients referred for suspected lung cancer: a quality improvement project to enhance patient safety in clinical practice. Lung Cancer 2020;139:S27.

7 Decroisette C, Monnet I, Berard H et al. Epidemiology and treatment costs of bone metastases from lung cancer: A French prospective, observational, multicenter study (GFPC 0601). Journal of Thoracic Oncology 2011;6:576-82.

Address for correspondence: Dr Christina Apthorp, Warwick Hospital, Lakin Road, Warwick, Warwickshire CV34 5BW, UK. Email: christina@apthorp.me.uk 